\title{
PHOTO ROUNDS
}

\section{Foot rash and joint pain}

\section{An untreated infection led to a series of unusual signs and symptoms that included difficulty walking.}

A 21-YEAR-OLD MAN presented to the emergency department (ED) with a 2-month history of joint pain, swelling, and difficulty walking that began with swelling of his right knee (FIGURE 1A). The patient said that over the course of several weeks, the swelling and joint pain spread to his left knee, followed by bilateral elbows and ankles. Nonsteroidal anti-inflammatory drugs (NSAIDs) and aspirin produced only modest improvement.

Two weeks prior to presentation, the patient also experienced widespread pruritus and conjunctivitis. His past medical history was significant for a sexual encounter that resulted in urinary tract infection (UTI)-like symptoms approximately 1 month prior to the onset of his joint symptoms. He did not seek care for the UTI-like symptoms.

In the ED, the patient was febrile $\left(102.1{ }^{\circ} \mathrm{F}\right)$ and tachycardic. Skin examination revealed erythematous papules, intact vesicles, and pustules with background hyperkeratosis and desquamation on his right foot (FIGURE 1B). The patient had spotty erythema on his palate and a 4-mm superficial erosion on the right penile shaft. Swelling and tenderness were noted over the elbows, knees, hands, and ankles. No inguinal lymphadenopathy was noted.

An arthrocentesis was performed on the right knee that demonstrated no organisms on Gram stain and a normal joint fluid cell count. A complete blood count (CBC), C-reactive protein (CRP), erythrocyte sedimentation rate (ESR), and urinalysis were ordered. A punch biopsy was performed on a scaly patch on the right elbow.

\section{O WHAT IS YOUR DIAGNOSIS? \\ O HOW WOULD YOU TREAT THIS PATIENT?}

Hannah R. Badon, MD; Ross L. Pearlman, MD; Robert T. Brodell, MD University of Mississippi Medical Center, Jackson

\section{arbrodell@umc.edu}

DEPARTMENT EDITOR Richard P. Usatine, MD University of Texas Health at San Antonio

The authors reported no potential conflict of interest relevant to this article.

doi: 10.12788/jfp.0199

\section{FIGURE 1}

\section{Swollen knee and foot lesions}
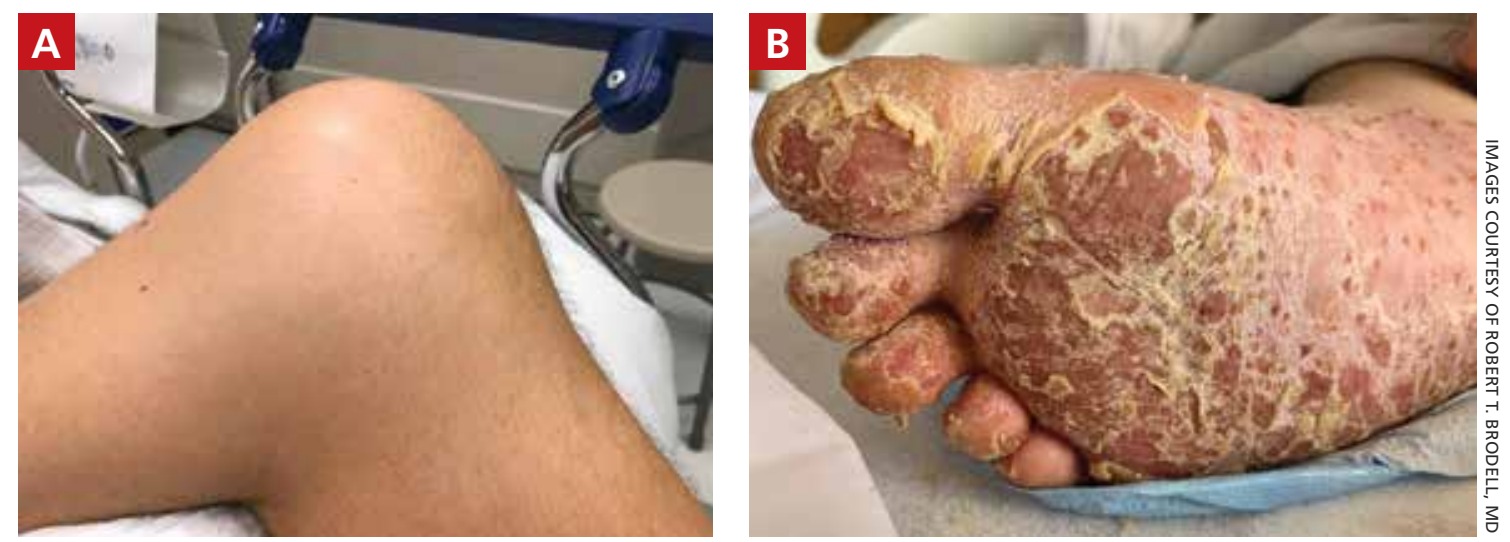

The 21-year-old patient presented with swelling over the right knee (A) and 1- to 3-mm papules on the sole of his right foot along with confluent thick scaling and desquamation (B). 


\section{$>$}

Reactive arthritis features a triad of conjunctivitis, urethritis, and arthritis that follows either gastrointestinal or urogenital infection.

\section{Dx: Keratoderma blenorrhagicum}

The patient's history, clinical findings, and lab results, including a positive Chlamydia trachomatis polymerase chain reaction (PCR) test from a urethral swab, pointed to a diagnosis of keratoderma blenorrhagicum in association with reactive arthritis (following infection with C trachomatis).

Relevant diagnostic findings included an elevated CRP of $26.5 \mathrm{mg} / \mathrm{L}$ (normal range, $<10 \mathrm{mg} / \mathrm{L}$ ), an elevated ESR of $116 \mathrm{~mm} / \mathrm{h}$ (normal range, $<15 \mathrm{~mm} / \mathrm{h}$ ) and as noted, a positive $C$ trachomatis PCR test. The patient's white blood cell count was 9.7/ $\mu \mathrm{L}$ (normal range, 4.5-11 $\mu \mathrm{L}$ ) and the rest of the CBC was within normal limits. Urinalysis was positive for leukocytes and rare bacteria. A treponemal antibody test was negative.

Additionally, the punch biopsy from the right elbow revealed acanthosis, intercellular spongiosis, and subcorneal pustules consistent with localized pustular psoriasis or keratoderma blenorrhagicum. After the diagnosis was made, human leukocyte antigen B27 allele (HLA-B27) testing was conducted and was positive.

\section{A predisposition \\ exacerbates the infection}

Reactive arthritis, a type of spondyloarthropathy, features a triad of conjunctivitis, urethritis, and arthritis that follows either gastrointestinal or urogenital infection. ${ }^{1}$ Reactive arthritis occurs with a male predominance of 3:1, and the worldwide prevalence is 1 in $3000 . .^{1}$ Causative bacteria include C trachomatis, Yersinia, Salmonella, Shigella, and Campylobacter, Escherichia coli, Clostridioides (formerly Clostridium) difficile, and $C$ pneumoniae. ${ }^{2}$ Patients with the HLA-B27 allele are 50 times more likely to develop reactive arthritis following infection with the aforementioned bacteria. ${ }^{1}$

Findings consistent with a diagnosis of reactive arthritis include a recent history of gastrointestinal or urogenital illness, joint pain, conjunctivitis, oral lesions, cutaneous changes, and genital lesions. ${ }^{3}$ Diagnostic tests should include arthrocentesis with cultures or PCR and cell count, ESR, CRP, CBC, and urinalysis. HLA-B27 can be used to support the diagnosis but is not routinely recommended. ${ }^{2}$

\section{Pustules and psoriasiform scaling characterize this diagnosis}

The differential diagnosis for the signs and symptoms seen in this patient include disseminated gonococcal arthritis, psoriatic arthritis, rheumatoid arthritis, and secondary syphilis.

I Gonococcal arthritis manifests with painful, sterile joints as well as pustules on the palms and soles, but not with the psoriasiform scaling and desquamation that was seen in this case. A culture or PCR from urethral discharge or pustules on the palms and soles could be used to confirm this diagnosis. ${ }^{3}$

I Psoriasis in association with psoriatic arthritis and the psoriasiform rashing of reactive arthritis (keratoderma blenorrhagicum) show similar histopathology; however, patients with psoriatic arthritis generally exhibit fewer constitutional symptoms. ${ }^{4}$

I Rheumatoid arthritis also manifests with joint pain and swelling, especially in the hands, wrists, and knees. This diagnosis was unlikely in this patient, where small joints were largely uninvolved. ${ }^{4}$

I Secondary syphilis also manifests with papular, scaly, erythematous lesions on the palms and soles along with pityriasis rosealike rashing on the trunk. However, it rarely produces pustules or hyperkeratotic keratoderma. ${ }^{5}$ As noted earlier, a treponemal antibody test in this patient was negative.

\section{Drug therapy}

\section{is the best option}

First-line therapy for reactive arthritis consists of NSAIDs. If the patient exhibits an inadequate response after a 2-week trial, intra-articular or systemic glucocorticoids may be considered. ${ }^{3}$ If the patient fails to respond to the steroids, disease-modifying antirheumatic drugs (DMARDs) may be considered. Reactive arthritis is considered chronic if the disease lasts longer than 6 months, at which point, DMARDs or tumor necrosis factor- $\alpha$ inhibitors may be utilized. ${ }^{3}$ For cutaneous manifestations, such as keratoderma blenorrhagicum, topical glucocorticoids twice daily may be used along with keratolytic agents.

I Our patient received 2 doses of azithromycin (500 mg IV) and 1 dose of ceftriaxone (2 g IV) to treat his infection while in the ED. 
Over the course of his hospital stay, he received ceftriaxone ( $1 \mathrm{~g}$ IV daily) for 6 days and naproxen (500 mg tid po) which was tapered. Additionally, he received a week of methylprednisolone (60 mg IM daily) before tapering to oral prednisone. His taper consisted of $40 \mathrm{mg}$ po for 1 week and was decreased by $10 \mathrm{mg}$ each week. Augmented betamethasone dipropionate $\mathbf{0 . 0 5 \%}$ cream and urea $20 \%$ cream were prescribed for twice-daily application for the hyperkeratotic scale on both of his feet.

\section{References}

1. Hayes KM, Hayes RJP, Turk MA, et al. Evolving patterns of reactive arthritis. Clin Rheumatol. 2019;38:2083-2088. doi: 10.1007/ s10067-019-04522-4

2. Duba AS, Mathew SD. The seronegative spondyloarthropathies. Prim Care. 2018;45:271-287. doi: 10.1016/j.pop.2018.02.005

3. Yu DT, van Tubergen A. Reactive arthritis. In: Joachim S, Romain PL, eds. UpToDate. Updated April 28, 2021. Accessed
June 3, 2021. https://www.uptodate.com/contents/reactivearthritis?search=reactive\%20arthritis\&topicRef $=5571 \&$ source $=$ see_link\#H9

4. Barth WF, Segal K. Reactive arthritis (Reiter's Syndrome). Am Fam Physician. 1999;60:499-503, 507.

5. Coleman E, Fiahlo A, Brateanu A. Secondary syphilis. Cleve Clin J Med. 2017;84:510-511. doi: 10.3949/ccjm.84a.16089

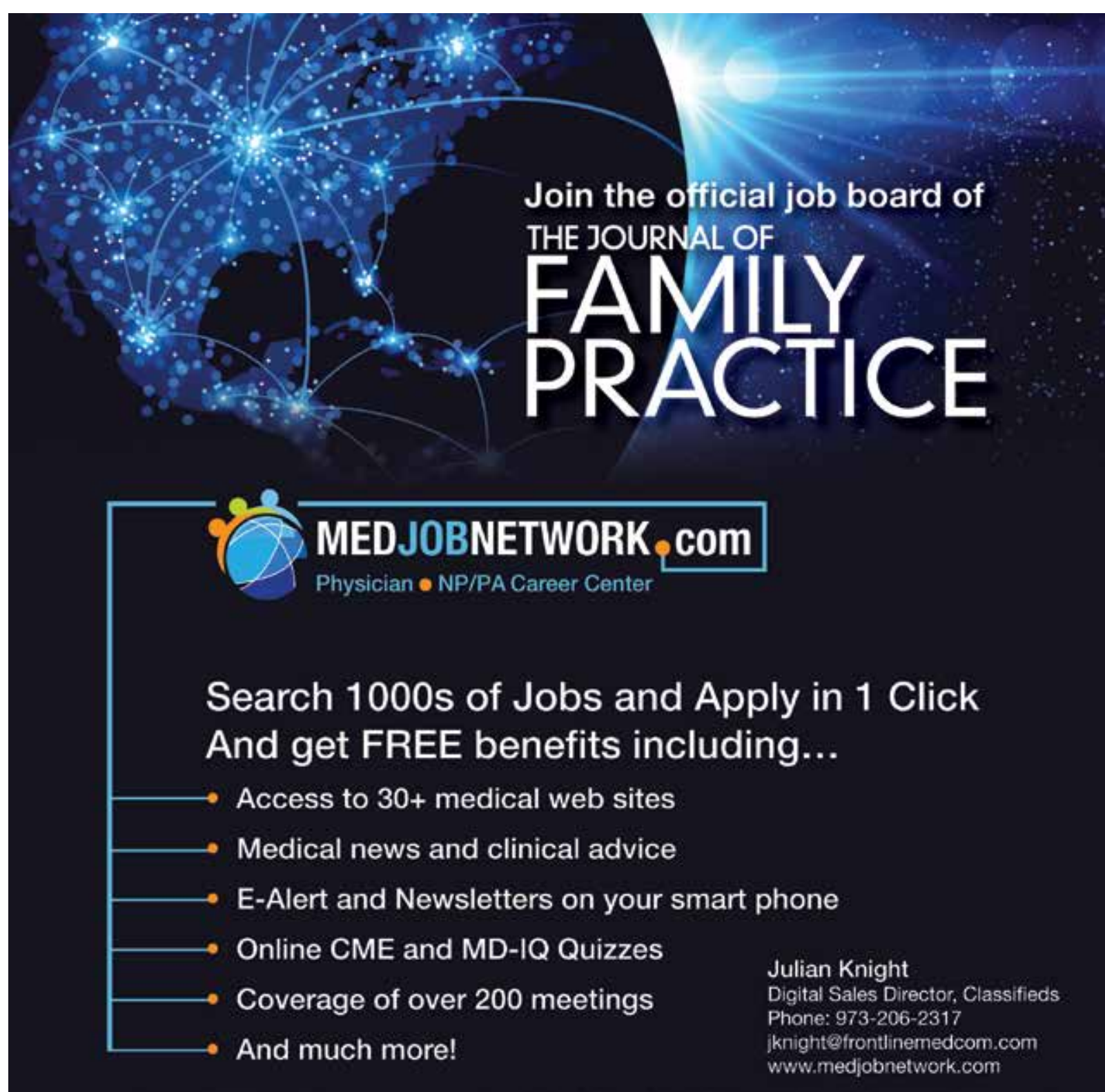

